\title{
Innovations
}

\section{Assessment for psychotherapy by interactive television suitable for transmission through telephone links}

\author{
P. M. McLaren, Research Psychiatrist, Academic Department of Psychiatry, \\ Guy's Hospital; C. J. BALL, Research Psychiatrist, Academic Department of \\ Psychiatry, Guy's Hospital; and J. P. WATson, Professor of Psychiatry, \\ Guy's Hospital, London SE1 9RT
}

Despite the increased availability of psychotherapy within the NHS, inequalities remain (Holmes \& Lindley, 1989). Traditionally, psychotherapy services have been provided from teaching centres in cities and patients have had to travel for treatment. This may account in part for the middle-class predominance in psychotherapy patients and Holmes (1991) has identified the need for psychotherapists to reach out and cater for the disadvantaged in large housing estates and rural areas. While services are changing to meet such needs (Pedder, 1989) there are powerful economic restrictions on service expansion. The potential for communications technology such as interactive television to aid the delivery of general psychiatric services has been reviewed by McLaren et al (1992) and similar arguments apply to the delivery of psychotherapy.

This study was performed as part of a project to use a new communications technology, computer based interactive television, to aid the delivery of psychiatric services including psychotherapy. The aim was to get a detailed description of professional user responses to using the system for assessing patients for psychotherapy as a preliminary to a clinical trial.

\section{The study}

Psychotherapy assessment on the LCVC

The communications equipment used was a prototype system known as the low cost videoconferencing system (LCVC). It is based on an Acorn Archimedes $310 \mathrm{M}$ (or 440) computer fitted with a Watford Archimedes Real-Time digitiser connected to a miniature monochrome video-camera. The LCVC software can be run in 1 Mbyte of memory and configured to load and run from disk when the power is switched on. A mouse based interface presents the video picture of the remote user in a quarter screen window (160 by 128 ) pixels on a monitor. Either 16 grey levels at 25 frames per second or 64 grey levels at 12.5 frames per second can be selected from an options menu. The miniature camera sits on top of the computer screen facing the user and the picture being sent to the person at the other end is shown on a second smaller monitor. A user seated four feet directly in front of the camera will fill the picture with head and shoulders. The digitised image produced by the system can be transmitted down 2 Megabit/ second telephone links. In this prototype system the sound was generated by a technics amplifier and loudspeaker system running in parallel to the videolink connection. The system connected two side rooms in adjacent psychiatric wards.

Two trained psychodynamic psychotherapists, who were also psychiatrists, were asked to assess ward patients, whom the responsible consultants thought might benefit from psychotherapy, by interviewing them on the LCVC. The patients were selected on the basis that they were not straightforward candidates for psychotherapy. The psychotherapists were informed that afterwards they would be questioned on their responses. The patients had both used the LCVC to talk to psychiatrists on several previous occasions and consented to be interviewed. The interactions were audiotaped.

\section{Observations}

Psychotherapist A saw a man of 27 years who had been admitted following an overdose of medication and whose repeated self-destructive behaviour had attracted negative reactions from nursing staff. The psychotherapist reported developing a negative reaction to the patient during the interview. Initially he began to feel frustrated by having difficulty hearing him. This was due to a combination of the patient mumbling and the technical limitations of the system. He reported that having to repeatedly ask the patient to speak up felt intrusive so he did not do it. He added that the patient's quiet voice and "the 
machine" created the effect of distance in the interaction. This was such that the psychotherapist felt the urge to "climb into the machine to get closer to the patient". When asked to interpret his feelings of frustration Psychotherapist A said he was uncertain whether this was a countertransference reaction to the patient or to "the machine". He also reported feeling inhibited in asking certain questions particularly on sexual topics and avoided these during the interview. Psychotherapist A reported that he sometimes felt uncomfortable talking on the telephone.

Psychotherapist B interviewed on the LCVC a 45year-old woman who had a paranoid illness with a circumscribed delusional system resistant to antipsychotic medication. He later described the interview as a "nice chat". He said he felt the LCVC undermined his ability to get "under the surface of the problem". He reported that he could no longer appreciate subtle non-verbal clues that he was "on the right track" with a line of questioning, such as dilatation of the pupils or a narrowing of the eyes. The use of his own body posture and positioning to influence the tone of the session was limited and he felt deprived of a valuable tool from his therapeutic armoury. He summed up the whole experience as "like trying to propose marriage to someone in Australia on the phone that you don't even know".

\section{Comments}

While only two patient-therapist dyads were studied the observations raise several important issues. The therapists had difficulty obtaining information from patients, interpreting their own responses and communicating nonverbally to the patients. In the use of communications technology for business activity, perceived interpersonal distance has been reported as an important variable in the interaction (Short et al, 1976). Therapist A described vividly such an effect when he reported wanting to "climb into the machine" to get closer to the patient. This therapist's negative response to LCVC may have generalised from feelings of discomfort experienced talking on the telephone. Such responses may be mediated by the perceived interpersonal distance, the lack of visual information for an individual who normally relies on it, or factors as yet unidentified. The uncertainty of $A$ as to the origins of their feelings of frustration is of interest. It could be attributed to the machine but the fact that other staff had similar feelings towards the patient seeing him face-to-face suggests it may have been a genuine response to the patient. Therapist $B$ also reported problems with the interview, and unequivocally attributed these to the machine, yet some of these difficulties may have also arisen in a face-to-face interview. It will be important for therapists to be aware of their own responses to the technology and to determine if, with repeated use, they can learn to dissect their responses to the patient from responses to the technology.

This pilot study of an interactive video system for a psychotherapeutic task illustrates the potential complexity of therapist responses. It points to some key concerns of psychotherapists' which will need to be addressed before the use of such equipment can be extended.

\section{Acknowledgements}

This Research was funded by the Telemed project (RACE-1086). Technical support was provided by BNR-Europe.

\section{References}

Holmes, J. (1991) Psychotherapy 2000: some predictions for the coming decade. British Journal of Psychiatry, 159, 149-155.

- \& LindLey, R. (1989) The Values of Psychotherapy. Oxford: Oxford University Press.

Mclaren, P. M., Watson, J. P., Summerfield, A. B. \& LIPSEDGE, M. (1992) Interactive television in psychiatry. Psychiatric Bulletin, 16, 288-291.

PEDDER, J. R. (1989) Courses in psychotherapy: evolution and current trends. British Journal of Psychotherapy, 6, 203-221.

Short, J. A., Williams, E. \& Christie, B. (1976) The Social Psychology of Telecommunications. London: Wiley International. 